\title{
Erythrophagocytosis in Bone Marrow: A Clue to Pyrexia of Unknown Origin
}

\author{
Neha Garg, Surabhi Raina, Mrinalini Kotru, Meera Sikka
}

Department of Pathology, University College of Medical Sciences and Guru Teg Bahadur Hospital, New Delhi, India

\begin{abstract}
Bone marrow (BM) is usually done to investigate the patients for Pyrexia of Unknown Origin (PUO). However, only $16.5 \%$ of cases reveal any diagnostic information. Increasing incidence of atypical presentations are seen in typhoid. Careful examination of BM to pick up clues for diagnosis is important We report a case of a 15 year old female who presented with fever since associated with altered sensorium, gum bleeding and loose stools. Hematological findings revealed pancytopenia with macrocytic anemia with erythrophagocytosis in BM aspirates. A diagnosis of typhoid was made with a positive typhi dot IgM. The authors present this case to highlight the importance of simple BM findings of erythrophagocytosis and how awareness about this feature can point towards the right diagnosis in the midst of atypical clinical and hematological features. J Microbiol Infect Dis 2018; 8(2):73-75.
\end{abstract}

Keywords: Bone marrow, typhoid fever, fever, macrophages, phagocytosis

\section{INTRODUCTION}

Bone marrow (BM) is usually done to investigate the patients for Pyrexia of Unknown Origin (PUO). However, only $16.5 \%$ of cases reveal any diagnostic information [1]. The authors present this case to highlight the importance of simple BM findings of erythrophagocytosis and how awareness about this feature can point towards the right diagnosis in the midst of atypical clinical and hematological features.

\section{CASE REPORT}

We report a case of a 15-year old female who presented with fever since 20-25 days associated with altered sensorium, gum bleeding and loose stools for 3-4 days. On examination, submandibular lymphadenopathy was present. No hepatosplenomegaly seen. Hemogram and peripheral smear showed pancytopenia (hemoglobin $4.7 \mathrm{~g} / \mathrm{dL}$, total leucocyte count $2,300 / \mathrm{mm}^{3}$, platelet count $15,000 / \mathrm{mm}^{3}$ ), macrocytic anemia along with toxic granulation of neutrophils. Bone Marrow Aspirates (BMA) were hypocellular, erythroid showing megaloblastic maturation with mild dyserythropoiesis and dysmyelopoiesis was seen. Histiocytes were increased with many showing hemophagocytosis particularly erythrophagocytosis (Figure 1). No granulomas seen. Bone Marrow Biopsy (BMB) showed similar features i.e. hemophagocytosis in the absence of granulomas. Hematological impression of cellular reactive marrow was given. A detailed work up for evaluating infective etiology was advised before considering primary hemophagocytosis. Malarial antigen (OptimalID) and widal test were negative. Blood and BM culture were not done. Typhi dot IgM (ELISA) came out to be positive. A diagnosis of typhoid was made. On follow up antibiotics were started and patient responded to treatment.

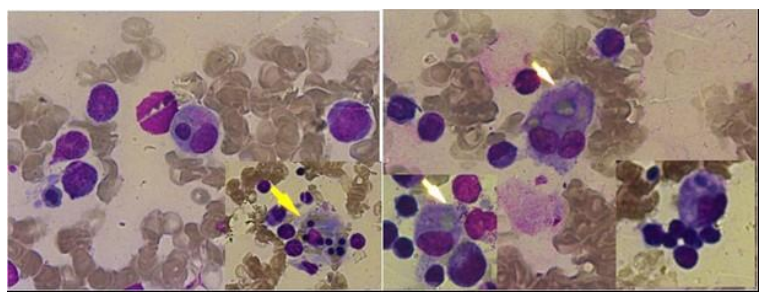

Fig 1. (right) MGG: Hemophagocytosis, 400X, (left): MGG: Erythrophagocytosis, 400X.

\section{DISCUSSION}

Tropical countries like India where typhoid fever is endemic, presentation as PUO can pose as a diagnostic dilemma. Increasing incidence of atypical presentations is quite often seen in endemic areas. In a study done by lqbal et al in Pondicherry, India $7 / 88(8 \%)$ of culture proven cases of typhoid fever presented as PUO [2]. Though, isolation of Salmonella typhi on blood culture remains the best method of diagnosis, it requires training of staff for specialized equipment, is expensive and takes time for incubation. Typhi dot is a simple, reliable and 
affordable rapid diagnostic test and is widely used for diagnosis of typhoid fever in developing countries like India.

Further, early institution of antibiotics may complicate the picture by altering the blood counts. The classic hematological abnormality seen in these patients is leucopenia with normohypercellular marrow due to myeloid hyperplasia with no dysplasia [2,3]. Our patient presented with pancytopenia with hypocellular BMA with dysplasia, all of which are infrequently reported hematological findings. Dutta et al reported BM depression in $2 / 32$ patients $(6.2 \%)$ of typhoid after ruling out other causes including antibiotics [4]. Our patient too was not receiving any antibiotics to cause BM depression. Dysmyelopoiesis in typhoid has been reported in a single case report till now [5]. In the present case, dysplasia in BMA could be attributed to associated megaloblastic anemia which has further complicated the hematological picture. But the presence of hemophagocytosis particularly erythrophagocytosis enlightened our way to correct diagnosis.

Typhoid fever is caused by Salmonella enterica. Bone marrow pathology in typhoid has been beautifully described by Shin et al [6]. The authors reported granulocytic hyperplasia, mild histiocytic proliferation with beginning of hemophagocytosis in early stage (up to 10 days from onset of symptoms), active hemophagocytosis (+ erythrophagocytosis) with beginning of the formation of ill-defined histiocytic granulomas in proliferative stage (1025 days from onset of symptoms), well defined epitheliod/histiocytic granulomas with erythrophagocytosis in late/lysis stage ( $>25$ days after onset of symptoms) [6]. Hence, presence of histiocytic proliferation with active erythrophagocytosis in the absence of granulomas in our patient may be due to the fact that patient was in third-fourth week of the illness corresponding to the proliferative stage.

Hemophagocytosis in typhoid is seen because this bacteria actively proliferates inside macrophages and stimulates them to hemophagocytose particularly non-senescent erythrocytes, the so called "typhoid cells" $[3,7]$. It has also been shown that macrophages infected by these bacteria are more likely to show erythrophagocytosis than non-infected macrophages [6].

Hemophagocytosis is frequently observed in Bacteria Associated Hemophagocytic Syndrome (BAHS) [6]. Rarely typhoid can also present as BAHS and few cases have been reported in literature $[6,8-10]$. In tropical countries like India where typhoid is endemic, hemophagocytic findings should be considered as Salmonella typhi associated BAHS until proved otherwise [6]. However, though serologically proven as typhoid, the present case did not meet the diagnostic criteria of BAHS [11].

Studies have reported the presence of erythrophagocytosis in typhoid along with BM Granuloma (BMG) [2,3,6] or rarely with BAHS6,8-10. There is only one study in literature which has reported typhoid presenting as erythrophagocytosis (typhoid cells) in the absence of BMG and BAHS. This study was done by Young et al in 1986 who has reported typhoid cells in $30 / 60 \quad(50 \%)$ patients. Coexistence with granuloma was seen only in four cases [12]. Various studies have suggested that typhoid fever should be considered in differential diagnosis of BMG especially in endemic areas next only to tuberculosis [3]. And more importantly, presence of erythrophagocytosis in a BMG is a clue towards the diagnosis of typhoid [3].

Increasing incidence of atypical presentations is seen in typhoid. Careful examination of BM to pick up clues for diagnosis is important. The present case highlights the importance of erythrophagocytosis in BM in detecting typhoid with atypical clinical and hematological features.

\section{ACKNOWLEDGMENTS}

Financial Support: None declared.

Conflict of interest: None declared.

\section{REFERENCES}

1) Gupta $R$, Setia $N$, Arora $P$, Singh $S$, Singh $T$. Hematological profile in pyrexia of unknown origin: role of bone marrow trephine biopsy vis-à-vis aspiration. Hematology. 2008;13(5):307-312

2) lqbal N, Basheer A, Mookkappan S, et al. Clinicopathological Profile of Salmonella Typhi and Paratyphi Infections Presenting as Fever of Unknown Origin in a Tropical Country. Mediterr J Hematol Infect Dis. 2015; 7(1):e2015021.

3) Muniraj K, Padhi S, Phansalkar M, Sivakumar P, Varghese RG, Kanungo R. Bone Marrow Granuloma in Typhoid Fever: A Morphological Approach and Literature Review. Case Rep Infect Dis 2015; 2015:628028.

4) Dutta TK, Beeresha, Ghotekar LH. Atypical manifestations of typhoid fever. J Postgrad Med 2001; 47(4):248-51

5) Chandra H, Chandra S, Gupta V, Mahajan D. Auer Rod in a Neutrophil in a Nonmalignant Condition. Turk J Haematol 2016; 33(2):167. 
6) Shin BM, Paik IK, Cho HI. Bone marrow pathology of culture proven typhoid fever. J Korean Med Sci 1994; 9(1):57-63.

7) Pilonieta MC, Moreland SM, English CN, Detweiler CS. Salmonella enterica infection stimulates macrophages to hemophagocytose. MBio 2014; 5(6):e02211-14.

8) Khalaf D, Toema B, Al-sadadi S, Al-jehani F, Sammak M. Salmonella Typhi Associated Hemophagocytic Lymphohistiocytosis in a Previously Healthy 23 Years Old Woman. Webmed Central Infect Dis 2011; 2(3):WMC001751.

9) Shah PA, Rashid A, Maqbool M, Yaseen Y, Shiekh FA. Enteric Fever Presenting as Hemophagocytic Lymphohistiocytosis (Macrophage Activation Syndrome). Webmed Central Infect Dis 2011; 2(10):WMC002331.

10) Chien YH, Lee PI, Huang LM, Lee CY, Lin DT, Lin $\mathrm{KH}$. Typhoid fever presenting as infection-associated hemophagocytic syndrome: report of one case. Acta Paediatr Taiwan. 1999; 40(5):339-340.

11) Douka E, Economidou F, Nanas S. Infections Associated with the Hemophagocytic Syndrome. Hosp Chron 2012; 7(1):16-24.

12) Jin SY, Lee KW, Kim TS. A study on the typhoid granuloma in the bone marrow biopsy of patients with typhoid fever. Korean J Pathol 1986; 20(4):462-469. 